\section{Characteristics of and risk factors for contact lens-related microbial keratitis in a tertiary referral hospital}

K Edwards ${ }^{1,2}$, L Keay ${ }^{1,2}$, T Naduvilath ${ }^{2}$, G Snibson ${ }^{3,4}, \mathrm{H}$ Taylor $^{2,3,4}$ and F Stapleton ${ }^{1,2}$

\section{Abstract}

Aim A retrospective case-control study was conducted at a tertiary referral hospital to determine the characteristics of and risk factors for contact lens (CL) related presumed microbial keratitis.

Methods Two hundred and ninety-one cases of presumed microbial keratitis were retrospectively identified over a 2-year period. Records were reviewed for a history of CL wear and, where identified, $\mathrm{CL}$, demographic, and clinical data were collected. Lens wearing controls $(n=186)$ were identified by a community telephone survey. Multiple logistic regression estimated risk factors for infection and vision loss.

Results Ninety-nine (34\%) new cases of presumed microbial keratitis were associated with $C L$ wear. Overnight soft $C L$ use was associated with an increased risk of infection compared to daily disposable CL wear (odds ratio (OR): $8.03,95 \%$ confidence interval $(\mathrm{CI})$ : 1.82-35.46). Compared with older CL wearers, 15-24 year olds had a 3.5 times greater risk of infection (OR, 95\% CI: 1.7-7.4). Of the 84 cases with available data, $24(29 \%)$ lost two or more lines of best-corrected visual acuity. Delaying treatment by $49-72 \mathrm{~h}$ had a 4.5 times (OR, 95\% CI: 1.4-14.9) greater risk of visual loss compared to seeking treatment early. Of the 99 cases of infection, 88 were scraped and $78 \%$ $(69 / 88)$ of these returned a positive culture. Gram-positive bacteria were the predominant causative organisms.

Conclusion Overnight use of CL and youth carry a greater risk of infection. Practitioners should reinforce the importance of proper CL care at all times, and early presentation following the onset of symptoms. Eye (2009) 23, 153-160; doi:10.1038/sj.eye.6702953; published online 17 August 2007
Keywords: keratitis, contact lenses, case-control studies

\section{Introduction}

Before the introduction of contact lenses, microbial keratitis (MK) was almost exclusively restricted to cases of trauma or ocular surface disease, and rarely occurred in healthy eyes. Depending on study design and location, contact lens wear now accounts for between 12.4 and $66 \%^{1-8}$ of all infective keratitis and up to $93 \%$ of all Acanthamoeba keratitis. ${ }^{9-11}$

To understand further and reduce the risk of infection, a number of large-scale epidemiological studies have investigated the risk factors for MK in contact lens wearers. Smoking ${ }^{12,13}$ poor lens and case hygiene, ${ }^{12-14}$ male gender, ${ }^{14}$ and lower socio-economic status ${ }^{1}$ are all associated with a higher risk of infection. Overnight use of lenses has been associated with a higher risk of MK as compared to daily wear in numerous studies across time and geographic location. ${ }^{13,15-17}$ The rate of continuous wear prescribing in Australia has increased from 1.6 to $11.7 \%$ over a 5-year period. ${ }^{18}$ As such, it is timely to reconsider the risk of MK with overnight use of lenses in Australia. We performed a retrospective, singlecentre case-control study to investigate the risk factors for contact lens-related MK and its associated vision loss in a hospital population.

\section{Methods}

\section{Subjects}

Contact lens wearers were defined as individuals wearing lenses for the correction of simple refractive errors aged between 15 and 64
${ }^{1}$ School of Optometry and Vision Science, The University of New South Wales, Sydney, Australia

${ }^{2}$ Vision Cooperative Research Centre, Sydney, Australia

${ }^{3}$ Centre for Eye Research Australia, The University of Melbourne, Melbourne, Australia

${ }^{4}$ Corneal Unit, Royal Victoria Eye and Ear Hospital, Melbourne, Australia

Correspondence: F Stapleton, School of Optometry and Vision Science, Level 3, North Wing, Rupert Myers Building, Gate 14, Barker Street, UNSW SYDNEY NSW 2052, Australia.

E-mail: f.stapleton@ unsw.edu.au

Received: 9 February 2007 Accepted in revised form: 17 July 2007 published online: 17 August 2007

Proprietary interests: The authors have no conflicting relationships or commercial connection to the topic of this paper. 
years, who had worn their lenses in the previous 4 weeks. Individuals wearing lenses for aphakia or keratoconus or for medical reasons were excluded from analysis. Lens wearers were placed into one of the four categories based on their lens material and actual mode of wear: rigid lenses (RGP), daily disposable lenses (DD), daily wear of soft lenses (DWS) and extended wear of soft lenses (EWS).

\section{Cases}

The Royal Victoria Eye and Ear Hospital (RVEEH) is a statewide teaching, training, and research tertiary referral hospital. All in-patient and casualty patient medical records from May 2001 to April 2003 with a relevant diagnosis were reviewed. In-patient files were reviewed with International Classification of Diseases 10 codes (H16.0 and H16.8), and emergency files were reviewed with relevant codes from the Victorian Emergency Minimum Dataset. Cases were included if the attending doctor documented, or if the treatment was consistent with, a diagnosis of microbial keratitis, and if there was a history of contact lens wear in the previous 4 weeks noted in the history. Ethics approval is not required for medical records reviews at the RVEEH; however, the Director of Medicine granted approval and the research was conducted in accordance with guidelines for confidentiality of medical records.

Data relating to age, gender, date of presentation, lens type, and mode of wear were collected. Clinical information was collated to give each individual a clinical severity score out of 10 (Table 1). The Index of Relative Socio-Economic Disadvantage (IRSD), derived from results of the Australian Bureau of Statistics 2001 Census, was used to determine the socio-economic status of each individual, based on postcode.

Vision loss of two lines or more was determined relative to vision in the fellow eye. Where fellow eye vision was not recorded or the eye was amblyopic, an acuity of $6 / 6$ was assigned.

Microbiological specimens were analysed by St Vincent's Health Pathology Services, located adjacent to the RVEEH. Methods for culturing samples have been described elsewhere. ${ }^{4}$ Sterile blades or needles were used to obtain samples, which were then smeared onto slides for Gram stain and onto culture media for isolation of bacteria, fungi, and Acanthamoeba. Samples were categorised as culture-negative, or revealing Grampositive bacteria, Gram-negative bacteria, fungus or Acanthamoeba species. Samples from which organisms were cultured on only one medium or only after extended incubation were assumed to be contaminated and were classified as culture-negative.

\section{Controls}

Controls were generated using a population-based telephone survey between October 2002 and June 2003. Households within the state of the hospital (Victoria) were selected randomly from the electronic White Pages. Before any telephone contact was attempted, households were sent a letter of introduction that provided study information and a toll free number for participants' questions. The telephone survey was approved by The University of New South Wales Human Research Ethics Committee and was conducted in accordance with the tenets of the Declaration of Helsinki, as revised in 2000.

Trained interviewers conducted telephone interviews between 1800 and 2100 hours, Monday to Thursday, and between 0900 and 1700 hours, Saturdays. Up to four attempts were made to establish contact with households, with each attempt made on a different day of the week or time of day. After establishing if there were lens wearers in the house, interviews were conducted with each lens wearer to establish details of lens type and mode of wear using a structured questionnaire. Further calls were made to interview all wearers if required.

\section{Statistical analysis}

Student's $t$-tests, one-way Analysis of Variance (ANOVA), Kruskal-Wallis test and $\chi^{2}$ analysis were used for bivariate analysis. Multiple logistic regression was used to estimate the determinants of risk of infection and risk of vision loss.

Model building using logistic regression followed standard methods including the investigation of

Table 1 Grading of severity out of 10; size of lesion was scored out of 3, location of lesion out of 3, and anterior chamber response out of 4

\begin{tabular}{|c|c|c|c|c|c|}
\hline & 0 & 1 & 2 & 3 & 4 \\
\hline Size ( $\mathrm{mm}$, longest dimension) & $<0.5$ & $0.5-1.0$ & $1.1-2.0$ & $>2$ & - \\
\hline Location & Peripheral & Mid-peripheral & $\begin{array}{c}\text { Occupying }>\text { two quadrants, or } \\
\text { with satellite lesion }\end{array}$ & Central & - \\
\hline Anterior chamber reaction & None & Endothelial dusting & Flare/cells & - & Hypopyon \\
\hline
\end{tabular}


confounding and interactions. Model discrimination was assessed using the area under receiver operating characteristic (ROC) curve. Statistical significance was investigated at the $95 \%$ level of confidence.

\section{Results}

\section{Cases and controls}

After review of 822 emergency and 94 in-patient records, 291 cases of MK were identified over the 2-year period. The excluded cases were either not related to lens wear, or were considered sterile keratitis. Ninety-nine (99; 34\%) of these cases were in current contact lens wearers. Information on lens type and mode of wear was available in 87 cases. Two hundred and sixty-five were identified in the phone survey. One hundred and eighty-six (186; $70.2 \%$ ) contact lens wearers from 184 households agreed to complete the survey.

\section{Risk factors for infection}

Bivariate analysis of cases and controls is shown in Table 2. Compared to controls, individuals with infections were significantly younger. Cases were more likely to be male, although this was not statistically significant. Presentation of cases was independent of season. EWS was associated with a higher risk of infection. Cases were over-represented in both the highest and lowest socio-economic quartiles.

Variables of significance of less than 0.50 were entered into a multivariate logistic regression model. Using multivariate analysis, risk factors for MK were overnight use of lenses, youth, and socio-economic status (Table 3 ).

\section{Risk factors for clinical severity}

Sufficient information on clinical severity (to assess a severity score) was available in 96 cases. Both lens type and severity score were able to be determined in 84 cases.

The severity of infection was associated with delay in seeking treatment, culture result, and history of travel (Table 4).

\section{Risk factors for vision loss}

Information on final visual outcome was available in 84 cases. Of the 15 cases where information was unavailable, seven were discharged to private care before event resolution, seven failed to attend final follow-up and one was not recorded in the notes. Of the 84 cases, $24(28.6 \%)$ had vision loss of at least two lines of bestcorrected visual acuity. Both lens type and visual outcome were available in 73 cases.
Table 2 Characteristics of cases and controls

\begin{tabular}{|c|c|c|c|}
\hline & $\begin{array}{c}\text { Cases } \\
(\mathrm{n}=99), \%\end{array}$ & $\begin{array}{c}\text { Controls } \\
(\mathrm{n}=186) \%\end{array}$ & P-value \\
\hline \multicolumn{4}{|l|}{ Age (years) } \\
\hline $45-64$ & 15.20 & 21.60 & 0.288 \\
\hline $35-44$ & 18.20 & 39.80 & Referent \\
\hline $25-34$ & 33.30 & 25.00 & 0.002 \\
\hline $15-24$ & 33.30 & 13.60 & $<0.001$ \\
\hline Male gender & 45.50 & 39.20 & 0.316 \\
\hline \multicolumn{4}{|l|}{ Lens type } \\
\hline DD & 4.60 & 9.70 & Referent \\
\hline RGP & 3.40 & 7.00 & 0.964 \\
\hline DWS & 67.80 & 77.40 & 0.286 \\
\hline EWS & 24.10 & 5.90 & 0.001 \\
\hline Season of presentation & & & 1.000 \\
\hline Summer & 24.20 & - & \\
\hline Autumn & 29.30 & & \\
\hline Winter & 23.20 & & \\
\hline Spring & 23.20 & & \\
\hline \multicolumn{4}{|l|}{$I R S D^{\mathrm{a}}$} \\
\hline 1st quartile (lowest) & 30.00 & 24.90 & 0.166 \\
\hline $2^{\text {nd }}$ quartile & 17.80 & 24.90 & Referent \\
\hline $3^{\text {rd }}$ quartile & 18.90 & 25.40 & 0.923 \\
\hline $4^{\text {th }}$ quartile (highest) & 33.30 & 24.90 & 0.092 \\
\hline \multicolumn{4}{|l|}{ Time to treatment ( $h$ ) } \\
\hline $0-24$ & 40.00 & - & \\
\hline $25-48$ & 13.70 & & \\
\hline $49-72$ & 21.10 & & \\
\hline$>72$ & 25.30 & & \\
\hline
\end{tabular}

Abbreviations: DD, daily disposable lenses; DWS, daily wear of soft lenses; EWS, extended wear of soft lenses; IRSD, The Index of Relative Socio-Economic Disadvantage; RGP, rigid lenses.

${ }^{a}$ All controls were ranked and divided into quartiles. Cases were then assigned into one of the four quartiles, based on the interquartile ranges of the controls. As such, if the IRSD distribution of the two groups were equal, it would be expected that each of the quartiles would contain $25 \%$ of the cases.

Table 4 shows bivariate analysis of risk factors for vision loss. Individuals who lost two or more lines of acuity were more likely to delay seeking treatment compared to those with no loss of visual acuity. Variables of significance of less than 0.50 were entered into a multivariate logistic regression model. Delay in seeking treatment was the only independent risk factor for vision loss; those who delayed seeking treatment by $49-72 \mathrm{~h}$ had a 4.50 times (odds Ratio (OR), 95\% confidence interval (CI): 1.36-14.94) greater risk of visual loss compared to those who sought treatment early (referent: 0-48 h after initial symptoms) (area under ROC curve $0.64(0.50-0.78)$ ). This did not hold for delays over $72 \mathrm{~h}$ (OR 1.07, 95\% CI: 0.28-4.00).

Severity of lesion and vision loss were strongly associated; lesions with no vision loss had an average 
Table 3 Multivariate analysis of risk factors for MK as determined by multiple binary logistic regression

\begin{tabular}{lc}
\hline & $M K$ \\
\hline $\begin{array}{l}\text { Area under ROC curve } \\
\text { Lens type }\end{array}$ & $0.73(0.67-0.80)$ \\
DD & Referent \\
RGP & $1.82(0.30-11.28)$ \\
DWS & $2.20(0.61-8.02)$ \\
EWS & $8.03(1.82-35.46)$ \\
& \\
Age (years) & Referent \\
$35-64$ & $2.54(1.29-5.00)$ \\
$25-34$ & $3.50(1.65-7.42)$ \\
15-24 & \\
Gender & NS \\
IRSD & \\
2nd and 3rd quartiles & Referent \\
1st quartile (lowest) & $1.63(0.81-3.27)$ \\
4 th quartile (highest) & $2.36(1.18-4.74)$ \\
\hline Abbreviations: DD, daily disposable lenses; DWS, daily wear of soft \\
lenses; EWS, extended wear of soft lenses; IRSD, The Index of Relative \\
Socio-Economic Disadvantage; NS, not significant; RGP, rigid lenses; \\
ROC, Receiver Operating Characteristic curve. \\
Values shown are odds ratios, with 95\% confidence intervals in \\
parenthesis.
\end{tabular}

severity score of $3.2 \pm 2.2$ while events with vision loss had a severity of $5.0 \pm 2.4(P=0.003)$.

\section{Microbiology}

Of the 99 cases of infection, 88 were scraped. Seventyeight percent $(69 / 88)$ of these returned a positive culture. Thirteen lesions were polymicrobial. There was no relationship between lens type and culture result $(P=0.32$; Table 5).

\section{Travel}

Of the 99 cases of infection, 17 had a history of recent travel; 11 were overseas visitors to Australia, three were visitors from interstate; and three were Australian residents with a history of recent overseas travel.

Compared to Australian patients, patients from overseas were significantly younger (8/11 aged 15-24 years, vs $25 / 88, P=0.03$ ). They also had different culture results to Australian patients; overseas patients had proportionally fewer Gram-positive lesions (1/11 vs $49 / 88, P=0.004)$ and proportionally more Gram-negative lesions $(6 / 11$ vs $13 / 99, P=0.006)$. Of the eight isolates of Pseudomonas aeruginosa, six were from overseas visitors (6/11 vs 2/88, $P<0.001)$.

Overseas travellers had significantly more severe lesions compared to Australian patients, although the
Table 4 Bivariate analysis of the characteristics affecting the severity of lesion and rate of vision loss

\begin{tabular}{|c|c|c|}
\hline & $\begin{array}{c}\text { Severity, } \mathrm{n}=96 \\
\text { (average severity) }\end{array}$ & $\begin{array}{c}\text { Vision loss, } \mathrm{n}=84 \\
\text { (prevalence of vision loss) }\end{array}$ \\
\hline Lens type & $P=0.195$ & \\
\hline RGP & & $66.7 \%(P=0.034)$ \\
\hline DD & & $33.3 \%(P=0.209)$ \\
\hline DWS & & $31.4 \%(P=0.074)$ \\
\hline EWS & & $6.3 \%$ (Referent) \\
\hline $\begin{array}{c}\text { Age (years) } \\
45-64 \\
35-44 \\
25-34 \\
15-24\end{array}$ & $P=0.336$ & $P=0.456$ \\
\hline Gender & $P=0.847$ & $P=0.809$ \\
\hline IRSD & $P=0.555$ & $P=0.826$ \\
\hline Season & $P=0.579$ & $P=0.761$ \\
\hline $\begin{array}{l}\text { All travel } \\
\text { Travellers } \\
\text { Non-travellers }\end{array}$ & $P=0.055$ & $P=1.000$ \\
\hline $\begin{array}{l}\text { Overseas travel } \\
\text { Overseas visitors } \\
\text { Local patients }\end{array}$ & $\begin{array}{c}P=0.024 \\
5.6 \pm 2.8 \\
3.5 \pm 2.5\end{array}$ & $P=0.710$ \\
\hline \multicolumn{3}{|c|}{ Time to treatment $(h)$} \\
\hline $0-48$ & $3.5 \pm 2.2(P=0.560)$ & $20.0 \%$ (referent) \\
\hline $49-72$ & $4.8 \pm 2.8(P=0.038)$ & $52.9 \%(P=0.014)$ \\
\hline$>72$ & $2.9 \pm 2.6$ (referent) & $21.1 \%(P=0.924)$ \\
\hline \multicolumn{3}{|l|}{ Microbiology } \\
\hline Not scraped & $2.7 \pm 2.3$ (referent) & $42.9 \%(P=0.350)$ \\
\hline Culture-negative & $3.7 \pm 2.4(P=0.703)$ & $23.5 \%$ (referent) \\
\hline $\begin{array}{l}\text { Gram-positive } \\
\text { bacteria }\end{array}$ & $3.2 \pm 2.4(P=0.917)$ & $23.3 \%(P=0.982)$ \\
\hline $\begin{array}{l}\text { Gram-negative } \\
\text { bacteria and } \\
\text { other }\end{array}$ & $5.8 \pm 2.6(P=0.001)$ & $41.2 \%(P=0.276)$ \\
\hline
\end{tabular}

Abbreviations: DD, daily disposable lenses; DWS, daily wear of soft lenses; EWS, extended wear of soft lenses; IRSD, The Index of Relative Socio-Economic Disadvantage; RGP, rigid lenses.

level of vision loss was similar between the two groups (Table 4). Delays in seeking treatment were similar between local and overseas patients $(P=0.40)$.

\section{Discussion}

The proportion of cases due to contact lens wear in this study is consistent with previous estimates. ${ }^{1-8,13,19} \mathrm{It}$ should be remembered that the age range here was restricted to 15-64 years and would, therefore, under- 
Table 5 Microbiology results for all lens types

\begin{tabular}{|c|c|c|c|c|c|c|}
\hline & All lenses $(\mathrm{n}=99)$ & $R G P(\mathrm{n}=3)$ & $D D(\mathrm{n}=4)$ & DWS $(\mathrm{n}=59)$ & $E W S(\mathrm{n}=21)$ & Lens unknown $(\mathrm{n}=12)$ \\
\hline \multicolumn{7}{|l|}{ Gram-positive cocci } \\
\hline Staphylococcus aureus & 2 & & & 2 & & \\
\hline Staphylococcus sp & 41 & 1 & 2 & 26 & 6 & 6 \\
\hline Streptococcus sp & 4 & & & 2 & 2 & \\
\hline Micrococcus sp & 2 & & & 2 & & \\
\hline Gram-positive cocci & 1 & & & & & 1 \\
\hline \multicolumn{7}{|l|}{ Gram-positive bacilli } \\
\hline Corynebacterium sp & 2 & & & 1 & 1 & \\
\hline Bacillus sp & 1 & & & 1 & & \\
\hline Propioni sp & 10 & & & 7 & 3 & \\
\hline Gram-positive rods & 1 & & & & & 1 \\
\hline Total Gram-positive & 64 & 1 & 2 & 41 & 12 & 8 \\
\hline \multicolumn{7}{|l|}{ Gram-negative bacilli } \\
\hline Pseudomonas aeruginosa & 8 & & 1 & 4 & 1 & 2 \\
\hline Serratia marcescens & 4 & 1 & & 3 & & \\
\hline Serratia liquefaciens & 2 & & & 2 & & \\
\hline Kingella kingae & 1 & & & 1 & & \\
\hline Gram-negative rods & 1 & & & & & 1 \\
\hline Total Gram-negative & 16 & 1 & 1 & 10 & 1 & 3 \\
\hline Acanthamoeba & 3 & 1 & & 2 & & \\
\hline Culture-negative & 19 & 1 & 1 & 10 & 6 & 1 \\
\hline Not scraped & 11 & & & 5 & 3 & 3 \\
\hline
\end{tabular}

Abbreviations: DD, daily disposable lenses; DWS, daily wear of soft lenses; EWS, extended wear of soft lenses; RGP, rigid lenses.

represent other causes of MK in older individuals associated with ocular surface or systemic disease. The RVEEH is a tertiary referral hospital and cases here may also represent the more severe spectrum of disease.

Overnight use of contact lenses is a well-established risk factor for $\mathrm{MK},{ }^{13,15-17}$ and is again associated with an increase in risk in this population. However, previous studies were conducted before the release of silicone hydrogel lenses, and this is one of the first studies considering the risk of infection in a population using this new lens type. Owing to the method of determining lens type from review of hospital notes, we were unable to consider silicone hydrogel lenses in isolation from traditional hydrogel materials in this study. Given that we know the majority of contemporary extended wearers use silicone hydrogel lenses, and the majority of daily wearers use traditional hydrogel materials, it could be interpreted that lens material, that is, silicone hydrogel lenses, are responsible for the increase in risk, rather than extended wear. However, given the higher risk

historically with overnight use of lenses, irrespective of lens type, and the advantages shown with silicone hydrogel lenses in laboratory and other clinical studies, this is extremely unlikely. Recent studies in the United Kingdom and Australia have found an increase in the risk of infection with (extended wear) EW of silicone hydrogel lenses compared to (daily wear) DW of hydrogel lenses. ${ }^{20-22}$ Seemingly, irrespective of lens type, overnight use of lenses still carries a higher risk of infection.

In this population, the risk of MK with DD lenses appears almost equivalent to that with RGP lenses. This is one of the first studies to consider the risk of infection with this new lens type, and it appears to confirm the hypothesis that DD lenses would carry a lower risk of infection. In interpreting this data, it should be considered that DD lenses tend to be used on a more 'part-time' basis compared to other lens types. Reduced exposure may reduce the risk; however, this should be confirmed in a prospective study, designed to consider such a factor.

These data indicate that youth is a risk factor for infection. Previous studies have found a similar association for sterile keratitis. ${ }^{14,23}$ This association was attributed to the higher prevalence of smoking in the younger age groups with infections; however, smoking was not able to be considered in this retrospective study. The higher risk of infection with youth was independent of lens type and socio-economic status (IRSD).

High socioeconomic status persists in this study, even after controlling in the statistical analysis for lens type and mode of wear. Similarly, preliminary analysis of a prospective study of MK using a similar indirect method of evaluating socioeconomic status has shown an 
unexpectedly higher risk of infection with higher socioeconomic status. ${ }^{22}$ Studies of cataract surgery in the United States have also found a U-shaped distribution in socio-economic status with the risk of disease. ${ }^{24}$ This was attributed to the greater accessibility of health care for those of higher socio-economic status. However, access to health care in Australia for an emergency condition such as MK should be independent of socio-economic status. As such, there may be other characteristics for those of higher status, such as compliance that were not considered here. Postcode-based classifications of socioeconomic status are not as accurate as those at the collection district level, ${ }^{25}$ which is acknowledged as a limitation of this study. It would have been more accurate to assess socio-economic status by directly asking patients about their occupation, level of education or income. However, this was not possible in a retrospective study.

Male gender was not a risk factor for infection. However, men did appear to be over-represented in the wearers with infections as compared to the control wearers, and previous studies have found such an association to be significant. ${ }^{1,15}$ These studies have only found an increased risk for men using DWS lenses, suggesting an association between lens hygiene and male gender. Indeed here, men were under-represented in the cases for soft lens EW (63.6\% of controls vs $47.6 \%$ of cases) and over-represented in the cases for soft lenses DW (36.1\% of controls vs $49.2 \%$ of cases).

The relationship found between clinical severity of lesions and the culture result is not surprising. Gramnegative bacteria have been found to be associated with larger lesions, ${ }^{16}$ and severe anterior chamber reaction. ${ }^{2}$ This also explains the relatively more severe lesions seen in overseas visitors, with the greater number of Pseudomonas infections.

The rate of vision loss of $28.6 \%$ is generally in agreement with previous studies.,16 This is likely to be higher than that found in the general community, as this study was at a tertiary referral hospital. Using the fellow eye as a referent, or 6/6 in the event of missing data, may have also artificially increased the rate of vision loss.

An association between delay in appropriate treatment and poor outcome is not a novel finding. ${ }^{26}$ A delay of 49-72 h in seeking treatment was the only significant risk factor for vision loss in this study. Surprisingly, this trend did not hold for delays over $72 \mathrm{~h}$. This may be because less severe events are associated with less severe symptoms, allowing individuals to tolerate symptoms for longer, a theory put forward in a recent study of corneal infiltrates. ${ }^{27}$ The microbiological profile of cases here supports this theory; of the 24 cases that took greater than $72 \mathrm{~h}$ to seek treatment, 19 cultured Gram-positive bacteria, one was Acanthamoeba keratitis, and none cultured Gram-negative bacteria.

A culture-positive rate of $78 \%$ is slightly higher than in similar hospital-based studies recently published. ${ }^{2,6}$ This may be a reflection of more severe lesions seen at a tertiary referral centre, or of different culturing practices in different hospitals.

Gram-negative bacteria traditionally have been the predominant causative organisms in contact lens-related infections; Pseudomonas species have been the most commonly isolated organism, ${ }^{13,16}$ sometimes accounting for over half of all isolates..$^{5,28,29}$ However, most of the infections in this study were associated with Grampositive bacteria. There are significant regional variations in the microbiological profile of contact lens-related infections. Gram-negative bacterial and fungal keratitis are more common in tropical climates, whereas keratitis associated with Gram-positive bacteria is more common in temperate climates. ${ }^{13}$ The microbiological profile of this dataset and the climate of Melbourne are consistent with this trend, as is a recent study of bacterial keratitis in France, where $69 \%$ of infections in contact lens wearers were associated with Gram-positive bacteria. ${ }^{2}$

An unexpected finding in this study was the high number of cases with a recent history of travel. While the equivalent information is not available for the controls, on Census night, 2001 (www.abs.gov.au, Census 2001), of the Victorian population, $0.7 \%$ were overseas visitors and $0.6 \%$ were interstate visitors. This is a very crude comparison, and does not take into account other factors, such as higher socio-economic status of those able to afford overseas travel and, hence, more likely able to afford contact lenses, or the possibility that travellers are more likely to seek medical treatment at hospitals rather than private clinics.

Owing to the retrospective nature of this study, a number of variables could not be included for analysis that previous studies have found to be risk factors for infection. As review of records were relied upon for information, we were not able to ascertain specific details on lens type and vision loss in a number of cases, reducing the power of our sample. Also, the number of records that did not include information on lens type $(12 / 99)$ could be a considerable flaw in our study if one particular lens type was over- or under-represented in the 12 . However, we have no reason to believe that such a bias exists.

Another possible limitation of this study is the noncontemporaneous generation of controls; cases presented from May 2001 to April 2003, while controls were contacted from October 2002 to June 2003. The prescribing of EW lenses has gradually increased in recent years. ${ }^{18}$ This discrepancy in timing may have caused a relative over-estimation of the number of 
extended lens wearers in the controls, causing an underestimation of the risk of MK with overnight use of lenses.

The selection of appropriate controls is often a difficult consideration in case-control studies. As the hospital here was a statewide hospital, controls were randomly selected from around the state. Although more severe cases of infection may be referred to RVEEH, this may not be true of mild cases. As such, the methods used to generate controls are appropriate for severe cases of infection, but less so for the milder cases.

\section{Conclusion}

This study provides one of the first insights into the risk of infection with DD and EW lenses in Australia. Although the potential limitations of this study should be kept in mind, overnight use of lenses carries a greater risk of infection. Patients should be reminded of the importance of proper lens care at all times. The importance of timely presentation in the case of adverse symptoms should also be stressed.

\section{Acknowledgements}

We acknowledge the clinical staff at RVEEH for ophthalmic care of study patients. We also acknowledge Kelly Lyngcoln and the medical records staff at RVEEH for facilitating the medical records review. This research was funded in part by the Australian Government via the Cooperative Research Centres Program, Canberra, Australia, and via a National Health and Medical Research Council (Canberra, Australia) Post-graduate Research Scholarship.

\section{References}

1 Dart JKG, Stapleton F, Minassian D. Contact lenses and other risk factors in microbial keratitis. Lancet 1991; 338(8768): 650-653.

2 Bourcier T, Thomas F, Borderie V, Chaumeil C, Laroche L. Bacterial keratitis: predisposing factors, clinical and microbiological review of 300 cases. Br J Ophthalmol 2003; 87(7): 834-838.

3 Fong CF, Tseng CH, Hu FR, Wang IJ, Chen WL, Hou YC. Clinical characteristics of microbial keratitis in a university hospital in Taiwan. Am J Ophthalmol 2004; 137(2): 329-336.

4 Keay L, Edwards K, Naduvilath T, Taylor HR, Snibson GR, Forde $\mathrm{K}$, et al. Microbial keratitis: predisposing factors and morbidity. Ophthalmology 2006; 113(1): 109-116.

5 Mela EK, Giannelou IP, Koliopoulos JX, Gartaganis SP. Ulcerative keratitis in contact lens wearers. Eye Cont Lens: Sci Clin Practice 2003; 29(4): 207-209.

6 Wong T, Ormonde S, Gamble G, McGhee CN. Severe infective keratitis leading to hospital admission in New Zealand. Br J Ophthalmol 2003; 87(9): 1103-1108.
7 Gebauer A, McGhee CN, Crawford GJ. Severe microbial keratitis in temperate and tropical Western Australia. Eye 1996; 10(Part 5): 575-580.

8 Rattanatam T, Heng WJ, Rapuano CJ, Laibson PR, Cohen EJ. Trends in contact lens-related corneal ulcers. Cornea 2001; 20(3): 290-294.

9 Radford CF, Lehmann OJ, Dart JK. Acanthamoeba keratitis: multicentre survey in England 1992-6. National Acanthamoeba Keratitis Study Group. Br J Ophthalmol 1998; 82(12): 1387-1392.

10 Radford CF, Minassian DC, Dart JKG. Acanthamoeba keratitis in England and Wales: incidence, outcome, and risk factors. Br J Ophthalmol 2002; 86(5): 536-542.

11 Stehr-Green JK, Bailey TM, Visvesvara GS. The epidemiology of Acanthamoeba keratitis in the United States. Am J Ophthalmol 1989; 107(4): 331-336.

12 Schein OD, Glynn RJ, Poggio EC, Seddon JM, Kenyon KR. The relative risk of ulcerative keratitis among users of dailywear and extended-wear soft contact lenses. A case-control study. Microbial Keratitis Study Group. New Engl J Med 1989; 321(12): 773-778.

13 Lam DS, Houang E, Fan DS, , Seal D, Wong E. Incidence and risk factors for microbial keratitis in Hong Kong: comparison with Europe and North America. Eye 2002; 16(5): 608-618.

14 Stapleton F, Dart JK, Minassian D. Risk factors with contact lens related suppurative keratitis. CLAO J 1993; 19(4): 204-210.

15 Poggio EC, Glynn RJ, Schein OD, Shannon MJ, Scardino VA et al. The incidence of ulcerative keratitis among users of daily-wear and extended-wear soft contact lenses. New Engl J Med 1989; 321(12): 779-783.

16 Cheng KH, Leung SL, Hoekman HW, Beekhuis WH, Mulder PG, Geerards AJ et al. Incidence of contact-lensassociated microbial keratitis and its related morbidity. Lancet 1999; 354(9174): 181-185.

17 Nilsson SE, Montan PG. The annualized incidence of contact lens induced keratitis in Sweden and its relation to lens type and wear schedule: results of a 3-month prospective study. CLAO J 1994; 20(4): 225-230.

18 Woods CA, Morgan PB. Use of silicone hydrogel contact lenses by Australian optometrists. Clin Exp Optom 2004; 87(1): 19-23.

19 Schein OD, Ormerod LD, Barraquer E, Alfonso E, Egan KM, Paton BG et al. Microbiology of contact lens-related keratitis. Cornea 1989; 8(4): 281-285.

20 Morgan PB, Efron N, Hill EA, Raynor MK, Whiting MA, Tullo AB. Incidence of keratitis of varying severity among contact lens wearers. Br J Ophthalmol 2005; 89(4): 430-436.

21 Radford CF, Stapleton F, Verma S, Minassian DC, Dart JKG. Microbial keratitis among users of silicone hydrogel contact lenses: interim analysis of a case control study. Invest Ophthalmol Vis Sci 2006; 47: B280. Abstract No.5564.

22 Stapleton F, Edwards K, Keay L, Naduvilath T, Dart JKG, Brian $\mathrm{G}$ et al. The incidence of contact lens related microbial keratitis in Australia. Invest Ophthalmol Vis Sci 2005; 46: B228. Abstract No.5025.

23 McNally JJ, Chalmers RL, McKenney CD, Robirds S. Risk factors for corneal infiltrative events with 30-night continuous wear of silicone hydrogel lenses. Eye Cont Lens: Sci Clin Practice 2003; 29(1 Suppl): S153-S156.

24 Klein R, Klein BE, Jensen SC, Moss SE, Cruickshanks KJ. The relation of socioeconomic factors to age-related cataract, 
maculopathy, and impaired vision. The Beaver Dam Eye Study. Ophthalmology 1994; 101(12): 1969-1979.

25 Hyndman JC, Holman CD, Hockey RL, Donovan RJ, Corti B, Rivera J. Misclassification of social disadvantage based on geographical areas: comparison of postcode and collector's district analyses. Int J Epidemiol 1995; 24(1): 165-176.

26 Miedziak AI, Miller MR, Rapuano CJ, Laibson PR, Cohen EJ. Risk factors in microbial keratitis leading to penetrating keratoplasty. Ophthalmology 1999; 106(6): $1166-1170$
27 Morgan PB, Efron N, Brennan NA, Hill EA, Raynor MK, Tullo AB. Risk factors for the development of corneal infiltrative events associated with contact lens wear. Invest Ophthalmol Vis Sci 2005; 46(9): 3136-3143.

28 Sharma S, Gopalakrishnan S, Aasuri MK, Garg P, Rao GN. Trends in contact lens-associated microbial keratitis in Southern India. Ophthalmology 2003; 110(1): 138-143.

29 Dejaco-Ruhswurm I, Scholz U, Hanselmayer G, Skorpik C. Contact lens induced keratitis associated with contact lens wear. Acta Ophthalmologica Scand 2001; 79(5): 479-483. 